\title{
Device Applications of Metal-2D-Materials Interfaces A Short Review
}

\author{
Faisal Ahmad, Amir Mansoori, Sonia Bansal, Th. S. Dhahi, and Shamim Ahmad
}

\begin{abstract}
The electronic energy band gaps of 2D-materials are known to spread over a wide range from zero in graphene to $>6 \mathrm{eV}$ in hexagonal boron nitride (h-BN). Various combinations of such engineered nanomaterials offer a number of novel device applications involving their unique optical, electronic, and thermal properties along with their higher charge carrier mobilities and saturation limited drift velocities. Structurally, these nanomaterials have single or multiple monolayers stuck together, which are not only suitable for flexible electron devices and circuits but also in preparing heterostructures (lateral as well as vertical configurations) that form super lattices with different kinds of band alignments. Such possibilities offer flexible control over the charge carrier transport in these materials via numerous types of exciton formations. Their extra sensitivity towards the presence of atomic, molecular and nanoparticulate species in their vicinity is the most significant aspect of these 2D-materials. This is the reason behind studying them in detail for detecting the presence of extremely low concentrations of the analyte that are not achievable in conventional sensors. For translating the above-said superlative properties of these fast emerging families of 2-D nanomaterials into usable devices and circuits, applying the conventional device fabrication technologies poses a real challenge. The experimental results reported in the context of forming usable interfaces between a metal and 2Dnanomaterial are examined here to assess their current status and future prospects. Their widespread applications are certainly anticipated in the fields like printed micro/nano sensors, large area electronics and printed intelligence with special reference to their emerging usages in Internet of Things (IoT) in the near future.
\end{abstract}

Index Terms-2D-Semiconductors; 2D-FET Structures; Edge Contacts; Fermi Level Pinning; Metal-Semiconductor Contacts; Planar Contacts; Schottky Barrier Heights.

\section{INTRODUCTION}

Every new semiconductor discovered is often characterized in terms of its temperature dependent conductivity, charge carrier mobility saturation limited drift velocity, carrier concentration, and optical transitions before exploring its possible applications in realizing a functional device with some characteristic properties. Having found suitable, the probable structural configurations are generally evolved using other materials in combination according to the requirements. While carrying out these exercises, choosing right kind of contacts to the input and output

Published on April 3, 2018.

F. Ahmad is with IRIS World Wide, Gurgaon, Haryana, India.

A. Mansoori, S. Bansal and S. Ahmad are associated with Physics Department, YMCA University of Science \& Technology, Faridabad, Haryana, India.

T. S. Dhahi hails from Physics Department, University of Basrah, Iraq.

Corresponding Author: S. Ahmad, drsahmad@email.com regions of the conceived device structure along with interconnect is critically important. In case the contact resistance of the often-used metal-semiconductor interface is bad, the device functions, although existing, might not be practically observed during measurement [1].

While conducting these experiments during early days of semiconductor device developments using conventional semiconductors, deposition of a metal thin film onto them as substrate invariably resulted in forming a Schottky contact due to the presence of a potential barrier depending upon the corresponding work function of the metal and the electron/hole affinity of the semiconductor. Higher values of the associated Schottky barrier height (SBH) across such contact interfaces lead to, in general, a rectifying contact. In case, $\mathrm{SBH}$ is close to zero or low, it produced an ohmic contact with linear I-V characteristics. This whole concept of metal-semiconductor contacts in conventional semiconductors has already been studied and extended to a number of combinations of wetting, barrier, and current carrying layers before employing them for fabricating a large variety of commercially produced discrete devices and IC's involving both ohmic and Schottky contacts [1].

Direct extension of the metal-semiconductor contact technology already matured adequately in the area of silicon and compound semiconductor devices and circuits is not that straightforward in case of 2D-semiconductors. The absence of the surface dangling bonds on either sides of such 2-D mono/multiple layers, and the prevailing anisotropic charge carrier transport across the active region give rise to very high contact resistance due to larger SBHs formed in addition to Fermi level pinning. Keeping these practical observations in view, even edge contacts were explored similar to those used in conventional HEMT devices that were developed up to commercial production level [1], [2].

Theoretically computed electronic structures of 2Dsemiconductors are found helpful in explaining the existence of such high contact resistances observed experimentally. Additionally, more theoretical investigations are still needed to study the influence of the existence of some external species in making the interface more susceptible to further changes. For example, applying quantum theoretical considerations, the minimum contact resistance is expected to be two orders of magnitude less than those observed in practice. This shows that there is still further scope for improving the device behavior involving these 2Dsemiconductors considerably [2].

Based on these observations made out of theoretical modeling and experimental measurements, attempts are made here in this paper to assess the current status and future prospects of forming high quality (i.e. low contact 
resistance) contacts/interconnects for their device applications in near future.

Realization of low contact resistance during fabrication of 2D mono/multiple layers based devices is essential for higher magnitude of $\mathrm{I}_{\mathrm{on}}$ current, better photo response, and high-frequency operation [3], [4]. The large contact resistance observed at the $2 \mathrm{D}$-mono/multiple layer - 3Dmetal interface, however, constrains the above-mentioned drain current [5] - [7]. Contacting 2D mono/multiple layers poses a number of experimental and conceptual problems. Theoretically, properties of these interfaces are decided by the chemical interactions taking place between the metal and the 2D-mono/multiple layers. The substitutional doping, a common method of reducing the contact resistance in common bulk semiconductors, is however not possible to extend here as it would modify both the 2D material and its interface properties. Further, the surfaces with no dangling bonds of a $2 \mathrm{D}$ mono/multiple layered materials make it difficult to form chemical bonds across the interface with a metal film, which is necessary to reduce the contact resistance. The quantum limit to the contact resistance determined by considering the number of allowed conducting modes within the channel, which is connected to the $2 \mathrm{D}$-charge carrier density, gives minimum $\mathrm{R}_{\mathrm{C}} \sim$ $30 \Omega . \mu \mathrm{m}$ at $\mathrm{n}_{2 \mathrm{D}}=10^{13} / \mathrm{cm}^{2}$, which is almost three orders of magnitude lower than the typical contact resistance established onto a monolayer $\mathrm{MoS}_{2}$ [8] - [10]. This clearly shows the necessity of further studies for improving the transport properties of the interface between metals and 2Dmono/multiple layers [2]. Some of the recent studies conducted in this direction are examined here in the followings to assess the current status before examining the future trend.

\section{Planar Contact Technology}

For realizing good ohmic contact between a metal and 2D-semiconductor, it is necessary to understand the corresponding charge carrier transport across such a 2Dsemiconductor - metal interface. To recapitulate the conventional model of metal-semiconductor junctions studied since long will certainly be helpful in this context [1]. For instance, in a conventional metal-semiconductor junction, the value of SBH decides whether it would be ohmic, or Schottky (i.e. rectifying) in nature. The SBH is defined as the difference between metal work function and the electron or hole affinities with respect to the conduction and valence band edges, respectively, in an $\mathrm{n}$ and $\mathrm{p}$-type semiconductor. Ideally, the alignment of the semiconductor's bands near the junction being ideally independent of the semiconductor's doping level, the $n$-type and $p$-type $\mathrm{SBH}$ ideally add to the band gap $\mathrm{E}_{\mathrm{G}}[1]$.

In case of significantly larger SBHs (i.e. barrier height $\left.\gg k_{B} T\right)$, the semiconductor next to the interface is invariably depleted creating a potential barrier. The charge carrier transport across such a potential barrier has already been modeled by thermionic emission across the barrier involving carriers within the thermal energy $k_{B} T$ around the energy equal to the barrier height [11]. In case of significantly lower SBHs, the semiconductor remains practically undepleted and thus offers ohmic contact behavior due to charge carrier tunneling. Under normal doping conditions, it is a mix of thermionic emission and tunneling of the charge carriers together that represent the resultant current-voltage characteristics [12]. Ideally, it is possible to reduce SBHs by doping the semiconductor to either $\mathrm{n}^{++}$or $\mathrm{p}^{++}$level (i.e. called heavy doping) favoring mostly charge carrier tunneling across the barrier.

However, under practical conditions, there are invariably significant deviations from these ideal conditions mentioned above, in brief, which ultimately give rise to interface states having their energies within the semiconductor band gap. Depending upon the distribution of these interface states across the band gap, the alignment of Fermi level gets clamped if sufficient density of states is there and form double layer in the semiconductor next to the filled surface states from the electrons supplied by the metal that screen the rest of the semiconductor from the influence of the metal work function. In such cases, the SBH becomes independent of the metal work function and this phenomenon is known as Fermi level (FL) pinning [13]. In general, the presence of interface states having energy levels within the band gap makes the whole situation quite complex. The role of these deviations will be referred back and forth while examining the cases of 2D-semiconductors as seen while discussing the specific cases.

The problems faced during integration of 2D-functional layers with three-dimensional (3D) systems were found limiting the device performances, and circuit designs comprising of the heterojunctions formed between 2D-3D materials as reviewed recently. For example, the contact resistance of metal on graphene and related 2D materials deserves a special attention, while the Schottky junctions formed between metal/2D semiconductor and graphene/3D semiconductor call for careful reconsideration of the physical models describing their junction behaviors. Several combinations of $2 \mathrm{D}$ and 3D- semiconductors $p-n$ junctions have currently been realized and characterized for their further applications [14].

Device designs of $\mathrm{MoS}_{2}$ based 2D-FETs were discussed on the basis of the understanding of the source/drain contacts for harvesting its intrinsic properties while finding optimum number of $\mathrm{MoS}_{2}$ layers. In this study using Scandium contacts on 10-nm-thick exfoliated $\mathrm{MoS}_{2}$ flakes covered with $15 \mathrm{~nm} \mathrm{Al}_{2} \mathrm{O}_{3}$ film demonstrated an effective mobility $\sim 700 \mathrm{~cm}^{2} / \mathrm{Vs}$ at room temperature. The confusion arising out of linear current-drain voltage characteristics was also clarified in this study [15].

$\mathrm{CNT}$ and $\mathrm{MoS}_{2}$ field-effect transistors were reported with asymmetric source-drain contacts, from which the influence of current directionality on contact resistances was estimated for the two current directions. By designing appropriate geometrical structures, it was concluded that the asymmetric electrical performance was caused by the difference in the effective SBH $\left(\Phi_{\mathrm{SB}}\right)$ caused by the different contacts. A detailed temperature-dependent study was, consequently, carried out to extract and compare the $\Phi_{\mathrm{SB}}$ for both the contact forms of CNT and $\mathrm{MoS}_{2}$ devices. It was noted that the $\Phi_{\mathrm{SB}}$ for the metal-on-semiconductor form was much lower than that of the semiconductor-on-metal form and was found suitable for all p-type, n-type, or ambipolar semiconductors. This observation was meaningful with respect to the design and applications of nanomaterial electronic devices. Additionally, using the difference in barrier height caused by the contact forms, Schottky barrier diodes were fabricated with a current ratio up to $10^{4}$. Rectifying circuits consisting of these diodes were able to 
work over a wide frequency range. This kind of design avoided the use of complex chemical doping or heterojunction formations in fabricating diodes that were relatively simple using only a single material. These findings might be suitably useful for future applications in nano electronic radio frequency devices, and integrated circuits [16].

is out of many 2D layered materials because of its unique properties that are useful in fabricating various electronic and optoelectronic devices with different band gap energies lying in the range of 1.29 to $1.9 \mathrm{eV}$ with decreasing number of layers involved. To control the number of $\mathrm{MoS}_{2}$ layers precisely, atomic layer etching (ALE) was used. It consisted of cyclic etching of a radical-adsorption step (e.g. Cladsorption) and a reacted-compound-desorption step via a low-energy $\mathrm{Ar}^{+}$-ion exposure. This was found effective in eliminating induced damages and contaminations that occurred during the reactive steps. Whereas, graphene is composed of one-atom-thick layers, $\mathrm{MoS}_{2}$ is composed of three-atom-thick $\mathrm{S}_{\text {(top) }}-\mathrm{Mo}_{\text {(mid) }}-\mathrm{S}_{\text {(bottom) }}$ layers; therefore, the ALE mechanisms of the two structures are significantly different. In this study, for $\mathrm{MoS}_{2} \mathrm{ALE}$, the $\mathrm{Cl}$ radical is used as the adsorption species and a low-energy $\mathrm{Ar}^{+}$ion is used as the desorption species. A $\mathrm{MoS}_{2}$ ALE mechanism (by which the $S_{(\text {top })}, M_{(\text {mid) }}$, and $S_{\text {(bottom) }}$ atoms were sequentially removed from the $\mathrm{MoS}_{2}$ crystal structure due to the trapped $\mathrm{Cl}$ atoms between the $\mathrm{S}_{(\text {top) }}$ layer and the $\mathrm{Mo}_{\text {(mid) }}$ layer) is reported according to the results of an experiment and a simulation study. In addition, the ALE technique showed that a monolayer $\mathrm{MoS}_{2}$ field effect transistor (FET) fabricated after one cycle of ALE was undamaged and exhibited electrical characteristics similar to those of a pristine monolayer $\mathrm{MoS}_{2}$ FETs. This technique was also found applicable to all layered TMD materials, such as tungsten disulfide $\left(\mathrm{WS}_{2}\right)$, molybdenum diselenide $\left(\mathrm{MoSe}_{2}\right)$, and tungsten diselenide $\left(\mathrm{WSe}_{2}\right)$ [17].

A simple method of fabricating TMD heterostructures was reported containing $\mathrm{MoSe}_{2} \mathrm{QDs}$ and a $\mathrm{MoS}_{2}$ or $\mathrm{WSe}_{2}$ monolayer. The strong modification of photoluminescence and Raman spectra that included the quenching of $\mathrm{MoSe}_{2}$ QDs and the varied spectral weights of trions for the $\mathrm{MoS}_{2}$ and $\mathrm{WSe}_{2}$ monolayers were observed, suggesting the occurrence of charge transfer in these TMD heterostructures. Such optically active heterostructures conveniently fabricated by dispersing TMD QDs onto TMD monolayers are likely to have various nano photonic applications because of their versatile and controllable properties [18].

The transport properties of a prototype multilayer $\mathrm{MoS}_{2} / \mathrm{WSe}_{2}$ hetero-junction were reported via forming a tunable charge inversion or depletion layer. This layer was prepared at the surface of $\mathrm{WSe}_{2}$ due to its relatively low doping concentration compared to that of $\mathrm{MoS}_{2}$, which could be tuned by the back-gate bias. The depletion region was limited within a few nanometers in the $\mathrm{MoS}_{2}$ side, while charges were fully depleted on the whole $\mathrm{WSe}_{2}$ side, which were confirmed by Raman spectroscopy and charge carrier transport measurements. The transport through the heterojunction was influenced by the presence of the inversion layer involving the two regimes of tunneling and recombination. Furthermore, photo-current measurements clearly revealed recombination and space-charge-limited behaviors, similar to those of the heterostructures built from organic semiconductors [19].
Band gap engineering of semiconductor heterojunctions for atomically thin opto-electronic devices was studied by preparing van der Waals heterostructures. With type II band alignment, interlayer excitons, where Coulomb bound electrons and holes were confined to opposite layers, have shown promising properties for novel excitonic devices, including a large binding energy, micron-scale in-plane drift-diffusion, and a long population and valley polarization lifetimes. The interlayer exciton optoelectronics based on electrostatically defined lateral $\mathrm{p}-\mathrm{n}$ junctions were demonstrated in a $\mathrm{MoSe}_{2}-\mathrm{WSe}_{2}$ hetero bi-layers. Applying a forward bias generated electro-luminescence from interlayer excitons. At zero bias, the $\mathrm{p}-\mathrm{n}$ junctions performed as highly sensitive photo detectors, where the wavelength-dependent photocurrent measurement allowed the direct observation of resonant optical excitation of the interlayer exciton. The resulting photocurrent amplitude from the interlayer exciton was $\sim 200$ times smaller than the resonant excitation of intra-layer exciton. This implied that the interlayer exciton oscillator strength was 2 orders of magnitude smaller than those of the intra-layer excitons due to the spatial separation of electron and hole to the opposite layers. These results formed the foundation for exploiting the interlayer excitons in future 2D-hetero-structure optoelectronic devices [20].

Fabrication of sub-10 nm gaps with sharp edges and steep sidewalls was reported as a novel technological method recently. It was found feasible to have sub-10 nm gaps from a corrosion crack along the cleavage plane of $\mathrm{Bi}_{2} \mathrm{O}_{3}$. By this method, ultrathin body field-effect transistors (FETs), consisting of $8.2 \mathrm{~nm}$ channel length, 6nm high-k dielectric, and $0.7 \mathrm{~nm}$ monolayer $\mathrm{MoS}_{2}$, exhibited no short channel effects. The corresponding current on/off ratio and sub threshold swing could reach up to $10^{6}$ and $140 \mathrm{mV} /$ decade, respectively. Moreover, integrated circuits with sub-10 $\mathrm{nm}$ channel devices operated as digital inverters with high voltage gain. These results suggested that this method could very well be used to fabricate the ultra-short channel nano patterns for the experimental groundwork for 2DMs FETs with sub-10 nm channel length and 2DMs integrated circuits [21].

Electrical metal contacts to two-dimensional (2D) semiconducting transition metal dichalcogenides (TMDCs) were found to be the major bottlenecks in fabricating devices due to strong Fermi level pinning and high contact resistances $\left(R_{\mathrm{c}}\right)$. Until now, Fermi level pinning of monolayer TMDCs has been reported only theoretically, although that of bulk TMDCs has been reported experimentally. An experimental study on Fermi level pinning of monolayer $\mathrm{MoS}_{2}$ and $\mathrm{MoTe}_{2}$ was reported by interpreting the thermionic emission results. In a recent work, the experimental measurements were compared with the theoretical simulation results of the monolayer structure as well as the experimental results of the bulk structure. The pinning factor was estimated to be 0.11 and -0.07 for monolayer $\mathrm{MoS}_{2}$ and $\mathrm{MoTe}_{2}$, respectively, suggesting a much stronger Fermi level pinning effect, a Schottky barrier height $(\mathrm{SBH})$ lower than that by theoretical prediction, and interestingly similar pinning energy levels between monolayer and bulk $\mathrm{MoS}_{2}$. These results further imply that metal work functions have very little influence on contact properties of 2D-material-based devices. Moreover, it was noted that $R_{\mathrm{c}}$ was exponentially proportional to $\mathrm{SBH}$, and these processing parameters could be controlled sensitively upon chemical doping into the 2D materials [22]. 
In search of an improved strategy for forming lowresistance contacts to semiconducting transition metal dichalcogenides, ab-initio DFT electronic structure calculations were carried out for $\mathrm{NbSe}_{2} / \mathrm{WSe}_{2}$ interface with quantum transport measurements of the corresponding hetero-junction between a few-layer $\mathrm{WSe}_{2}$ semiconductor and a metallic $\mathrm{NbSe}_{2}$ layer. The theoretical results suggested that, besides a rigid band shift associated with charge transfer the presence of $\mathrm{NbSe}_{2}$ did not modify the electronic structure of $\mathrm{WSe}_{2}$. Since the two transition metal dichalcogenides are structurally similar and display only a small lattice mismatch, their hetero-junction could efficiently transfer charge across the interface. These findings were supported by transport measurements for $\mathrm{WSe}_{2}$ field-effect transistors with $\mathrm{NbSe}_{2}$ contacts, which exhibited nearly ohmic behavior and phonon-limited mobility in the hole channel, indicating that the contacts to $\mathrm{WSe}_{2}$ were highly transparent [23].

In one of the experiments, graphene- $\mathrm{WS}_{2}$ heterojunctions were prepared over large areas using a seedless ambientpressure CVD technique. Techniques like Kelvin probe force microscopy, photo luminescence spectroscopy, and scanning tunneling microscopy were used to characterize the doping in graphene--WS $\mathrm{W}_{2}$ heterojunctions as-grown on sapphire and transferred to $\mathrm{SiO}_{2}$ with and without thermal annealing. Both $\mathrm{p}-\mathrm{n}$ and $\mathrm{n}-\mathrm{n}$ junctions were observed, and a flat-band condition (zero Schottky barrier height) was found for lightly $\mathrm{n}$-doped $\mathrm{WS}_{2}$, promising low-resistance ohmic contacts. This indicated a more favorable band alignment for graphene- $\mathrm{WS}_{2}$ than the theoretically predicted one, likely explaining the low barriers observed in transport experiments on similar heterojunctions. Simulations demonstrated that the large depletion width of the graphene$\mathrm{WS}_{2}$ junction reflected the electrostatics of the onedimensional junction between two-dimensional materials [24].

Contacts were made to stacked van der Waals (vdW) heterostructures by overlaid graphene electrodes enabling realization of atomically thin and flexible electronics. The first-principles quantum transport simulations of graphenecontacted $\mathrm{MoS}_{2}$ devices were developed to show the critical dependence of the transistor effect on the stacking configuration relative to the gate electrode. This could, thus, be traced to the stacking-dependent response of the contact region to the capacitive electric field induced by the gate. The contact resistance being a central parameter, these observations established important design rules for fabrication of ultrathin devices based on 2D atomic crystals [25].

In one of the studies carefully designed aqueous droplet light heating system was used along with a thorough mathematical procedure, which led to a precise determination of internal light-to-heat conversion efficiency of a variety of nanomaterials. The internal light-to-heat conversion efficiency of $\mathrm{Ti}_{3} \mathrm{C}_{2}$, was measured to be $100 \%$, indicating a perfect energy conversion. Furthermore, a selffloating MXene thin membrane was prepared by simple vacuum filtration and the membrane, in the presence of a rationally chosen heat barrier, produced a light-to-waterevaporation efficiency of $84 \%$ under one sun irradiation, which is among the state of art energy efficiency for similar photo thermal evaporation system. The outstanding internal light-to-heat conversion efficiency and great light-to-water evaporation efficiency reported in this work suggested that
MXene was a very promising light-to-heat conversion material deserving further explorations [26].

A novel MAX ceramic biomaterial was reported for the first time exhibiting the unique functionality for the photo thermal ablation of cancer upon being exfoliated into ultrathin nanosheets within atomic thickness (MXene). Biocompatible $\mathrm{Ti}_{3} \mathrm{C}_{2}$ nanosheets were synthesized based on a two-step exfoliation strategy of $\mathrm{Ti}_{3} \mathrm{AlC}_{2}$ by the combined $\mathrm{HF}$ etching and TPAOH intercalation. Especially, the high photo thermal-conversion efficiency and in-vitro/invivo photo thermal ablation of tumor of $\mathrm{Ti}_{3} \mathrm{C}_{2}$ nanosheets (MXenes) were revealed and demonstrated, not only in the intravenous administration of soybean phospholipid modified $\mathrm{Ti}_{3} \mathrm{C}_{2}$ nanosheets but also in the localized intratumoral implantation of a phase-changeable PLGA/Ti ${ }_{3} \mathrm{C}_{2}$ organic-inorganic hybrid. This study offered sufficient potentials of $\mathrm{Ti}_{3} \mathrm{C}_{2}$ nanosheets as a novel ceramic photo thermal agent used for cancer therapy [27].

A study reported about the contacts formed onto the surface of various metals and single-layer $\mathrm{MoSe}_{2}$. Partial Fermi level pinning was demonstrated by the first-principle calculations, which indicated modulation of the electron Schottky barrier. Upon inserting a $\mathrm{VS}_{2}$ layer between $\mathrm{MoSe}_{2}$ layer and metal electrodes, all the n-type contacts at $\mathrm{MoSe}_{2} /$ metal interfaces turned into p-type, and the hole Schottky barrier could be tuned effectively by varying metal electrodes. The high work function of the $\mathrm{VS}_{2}$ layer exerted significant influence on the band realignment of $\mathrm{MoSe}_{2}$, making all the n-type contacts at $\mathrm{MoSe}_{2} /$ metal interfaces become p-type contacts at $\mathrm{MoSe}_{2} / \mathrm{VS}_{2}-$ metal interfaces. Variation of the Schottky barriers and band alignments with the work function of metal electrodes demonstrated a partial Fermi level pinning at the interfaces of $\mathrm{MoSe}_{2} /$ metal and $\mathrm{MoSe}_{2} / \mathrm{VS}_{2}-$ metal. The partial Fermi level pinning resulted from the low density of interfacial states, which could be reflected partly by the interaction between $\mathrm{MoSe}_{2}$ layer and metal electrodes [28].

Monolayer $\mathrm{MoS}_{2}$ is a direct band gap semiconductor with a band gap of $1.8 \mathrm{eV}$. Recently, field-effect transistors were demonstrated using a mechanically exfoliated $\mathrm{MoS}_{2}$ monolayer, showing promising potential for next generation electronics. The ultimate performance limit of $\mathrm{MoS}_{2}$ transistors was estimated by using non equilibrium Green's function based quantum transport simulations showing that the strength of $\mathrm{MoS}_{2}$ transistors lies in large ON-OFF current ratio $\left(>10^{10}\right)$, immunity to short channel effects (drain-induced barrier lowering $\sim 10 \mathrm{mV} / \mathrm{V}$ ), and abrupt switching (sub-threshold swing as low as $60 \mathrm{mV} /$ decade). Our comparison of monolayer $\mathrm{MoS}_{2}$ transistors to the state-of-the-art III-V materials based transistors, reveals that while $\mathrm{MoS}_{2}$ transistors may not be ideal for high-performance applications due to heavier electron effective mass $\left(m^{*}=0.45 m_{o}\right)$ and a lower mobility, they can be an attractive alternative for low power applications thanks to the large band gap and the excellent electrostatic integrity inherent in a two-dimensional system [29].

Discrete electronic and optoelectronic components like FETs, sensors, and photo-detectors made from few-layer $\mathrm{MoS}_{2}$ showed promising performance as a substitute of $\mathrm{Si}$ in conventional electronics and of organic and amorphous $\mathrm{Si}$ semiconductors in ubiquitous systems and display applications. The next step is to fabricate fully integrated multistage circuits and logic building blocks on $\mathrm{MoS}_{2}$ to 
demonstrate its capability for complex digital logic and high-frequency ac applications. This was demonstrated in an inverter, NAND gate, static random access memory, and five-stage ring oscillator based on a direct-coupled transistor logic technology. The circuits consisted of 2 to 12 transistors seamlessly integrated side-by-side on a single sheet of bilayer $\mathrm{MoS}_{2}$. Both enhancement-mode and depletion-mode transistors were fabricated thanks to the use of gate metals with different work functions [30].

The electronic properties of graphene in contact with monolayer and bilayer $\mathrm{PtSe}_{2}$ were studied using firstprinciples calculations. It turned out that there is no charge transfer between the components because of the weak van der Waals interaction while calculating the work functions of monolayer and bilayer $\mathrm{PtSe}_{2}$ and analyzing the band bending at the contact with graphene. The formation of an ntype Schottky contact with monolayer $\mathrm{PtSe}_{2}$ and a p-type Schottky contact with bilayer $\mathrm{PtSe}_{2}$ was demonstrated. The Schottky barrier height was very low in the bilayer case and could be reduced to zero by $0.8 \%$ biaxial tensile strain [31].

Atomically thin 2D materials are difficult to fabricate without degradation of the original properties of the material required in realizing 2D-material devices. Atomic layer deposition (ALD) was found an ideal technique for adding materials with atomic scaling precision to nanomaterials. Due to the surface-sensitive reactions of ALD, growth on 2D materials is strongly affected by the surface properties of the 2D materials. In this Perspective, ALD growth on 2D materials is reviewed and discussed with previously reported results to provide insights for investigating $2 \mathrm{D}$ materials [32].

Atomically thin $\mathrm{MoS}_{2}$ is an ideal semiconductor material for FETs with sub-10 nm channel lengths. The high effective mass and large band gap of $\mathrm{MoS}_{2}$ minimize direct source-drain tunneling, while its atomically thin body maximizes the gate modulation efficiency in ultra-shortchannel transistors. However, no experimental study to date has approached the sub-10 nm scale due to the multiple challenges related to nanofabrication at this length scale and the high contact resistance traditionally observed in $\mathrm{MoS}_{2}$ transistors. Using semiconducting-to-metallic phase transition of $\mathrm{MoS}_{2}$, a recent study demonstrated sub-10 nm channel-length transistor fabricated by directed selfassembly patterning of mono and trilayer $\mathrm{MoS}_{2}$. This was done in a $7.5 \mathrm{~nm}$ half-pitch periodic chain of transistors where semiconducting $(2 \mathrm{H}) \mathrm{MoS}_{2}$ channel regions were seamlessly connected to metallic-phase $\left(1 \mathrm{~T}^{\prime}\right) \mathrm{MoS}_{2}$ access and contact regions. The resulting $7.5 \mathrm{~nm}$ channel-length $\mathrm{MoS}_{2}$ FET gave low off-current of $10 \mathrm{pA} / \mu \mathrm{m}$, an on/off current ratio of $>10^{7}$, and a sub threshold swing of 120 $\mathrm{mV} / \mathrm{dec}$ ade. The experimental results presented in this work, combined with device transport modeling, reveal the remarkable potential of $2 \mathrm{D} \mathrm{MoS}_{2}$ for future sub-10 nm technology nodes [33].

A novel approach was reported from a recent work for achieving low-resistance contacts to $\mathrm{MoS}_{2}$ transistors with the intrinsic performance of the $\mathrm{MoS}_{2}$ channel preserved. Through a dry transfer technique and a metal-catalyzed graphene treatment process, nickel-etched-graphene electrodes were fabricated on $\mathrm{MoS}_{2}$ that yielded contact resistance as low as $200 \Omega \cdot \mu \mathrm{m}$. The substantial contact enhancement ( $\sim 2$ orders of magnitude), as compared to pure nickel electrodes, was attributed to the much smaller work function of nickel-graphene electrodes, together with the fact that presence of zigzag edges in the treated graphene surface enhanced the tunneling between nickel and graphene. To this end, the successful fabrication of a clean graphene- $\mathrm{MoS}_{2}$ interface and a low resistance nickelgraphene interface was critical for the experimentally measured low contact resistance [34].

A new strategy was demonstrated by fabricating $2 \mathrm{D} / 2 \mathrm{D}$ low-resistance ohmic contacts for a variety of transition metal dichalcogenides (TMDs) using van der Waals assembly of substitutionally doped TMDs as drain/source contacts and TMDs with no intentional doping as channel materials. It could be demonstrated that few-layer $\mathrm{WSe}_{2}$ FETs with 2D/2D contacts exhibited low contact resistances of $\sim 0.3 \mathrm{k} \Omega . \mu \mathrm{m}$, high on $/$ off ratios $>10^{9}$, and high drive currents exceeding $320 \mu \mathrm{A} / \mu \mathrm{m}$. These characteristics were combined with a two-terminal field-effect hole mobility $\mu_{\mathrm{FE}} \sim 200 \mathrm{~cm}^{2} / \mathrm{Vs}$ at room temperature, which increases to $>2000 \mathrm{~cm}^{2} / \mathrm{Vs}$ at cryogenic temperatures. A similar performance was observed in $\mathrm{MoS}_{2}$ and $\mathrm{MoSe}_{2}$ FETs with 2D/2D drain and source contacts. The 2D/2D low-resistance ohmic contacts presented here represented a new device paradigm that could take care of a significant bottleneck in the performance of TMDs and a wide variety of other 2D materials as the channel materials in post silicon electronics [35].

The origin of the improved contact properties of alloyed 2D metal-semiconductor heterojunctions were studied in a recent work by fabricating $\mathrm{WSe}_{2}$ transistors with mixed transition layers containing van der Waals (M-vdW, $\mathrm{NbSe}_{2} / \mathrm{W}_{\mathrm{x}} \mathrm{Nb}_{1-\mathrm{x}} \mathrm{Se}_{2} / \mathrm{WSe}_{2}$ ) junctions realizing atomically sharp interfaces, exhibiting long hot-carrier lifetimes of approximately $\sim 75,296 \mathrm{~s} \quad(\sim 80$ times longer than that of metal-semiconductor, $\mathrm{Pd} / \mathrm{WSe}_{2}$ junctions). Such dramatic lifetime enhancement in $\mathrm{M}-\mathrm{vdW}$-junction devices was attributed to the synergistic effects arising from the significant reduction in the number of defects and the Schottky barrier lowering at the interface. Formation of a controllable mixed-composition alloyed layer on the 2D active channel could be a breakthrough approach to maximize the electrical reliability of 2D nanomaterial-based electronic applications [36].

A recent research study of $n$ - and p-type carrier injections was reported in case of $\mathrm{WSe}_{2}$ using van der Waals (vdW) contacts of two-dimensional (2D) materials: graphite for an n-type contact and $\mathrm{NbSe}_{2}$ for a p-type contact. Instead of conventional methods such as the evaporation of metals on TMD, 2D metals were transferred onto $\mathrm{WSe}_{2}$ in order to form van der Waals contacts. These contacts showed a small Schottky barrier height for both carrier polarities. These finding revealed the potential of a high-performance vdW metal/ semiconductor contact for use in electronics applications [37].

To overcome existing limitations in sensitivity and cost of state-of-the-art systems having high-sensitivity photo detection covering a large spectral range from the UV to IR is dominated by photodiodes, new device architectures and material systems are needed with low-cost fabrication and high performance. In the perspective of using low dimensional materials, the physical mechanism of photoFETs (field-effect transistors) was described with recent advances in the field of low-dimensional photo-FETs and hybrids. The requirements of the channel material were addressed in view of the photon absorption and carrier transport process, and a fundamental trade-off between them 
was pointed out for single-material-based devices. It was also clarified how hybrid devices, consisting of an ultrathin channel sensitized with strongly absorbing semiconductors, can circumvent these limitations and lead to a new generation of highly sensitive photo detectors. Recent advances in the development of sensitized low-dimensional photo-FETs were discussed along with several promising future directions for their application in high-sensitivity photo detection [38].

The extensive energy band calculation schemes fail to reproduce the observed SBHs in $2 \mathrm{D} \mathrm{MoS}_{2}-\mathrm{Sc}$ interface. An $\mathrm{ab}$ initio quantum transport device simulation better reproduces the observed $\mathrm{SBH}$ in $2 \mathrm{D} \mathrm{MoS}_{2}-\mathrm{Sc}$ interface and highlights the importance of a higher level theoretical approach beyond the energy band calculation in the interface study. BL $\mathrm{MoS}_{2}$-metal contacts generally have a reduced $\mathrm{SBH}$ than $\mathrm{ML} \mathrm{MoS}_{2}$-metal contacts due to the interlayer coupling and thus have higher electron injection efficiency. A theoretical study was conducted comparing the interfacial properties of monolayer and bilayer $\mathrm{MoS}_{2}$ on Sc, Ti, Ag, Pt, Ni, and Au by using different theoretical models. Comparing the calculated and observed Schottky barrier heights suggested that many-electron effects are strongly depressed and the transport gap of a device depends on the DFT-GGA rather than the quasi particle band gap. Such a depression of many-electron effects can be applied to a general metal-2D semiconductor interface. In generally, the Schottky barrier heights are decreased from $\mathrm{ML} \mathrm{MoS}_{2}$-metal interfaces to $\mathrm{BL} \mathrm{MoS}_{2}$-metal interfaces due to the interlayer coupling, implying that $\mathrm{BL} \mathrm{MoS}_{2}$ with a higher electron injection efficiency is probably more suitable for a transistor than $\mathrm{ML} \mathrm{MoS}_{2}$ given the same gate controllability [39].

The problem in the understanding of the metal-2D semiconductor interface primarily comes from the anisotropy of the in-plane and out-of-plane electrical conductivity of the 2D-semiconductor. In-plane conductivity is almost two orders of magnitude higher than the out-ofplane conduction, which may severely affect the charge transport into the 2D semiconductors. For studying the importance of these two conductivity components two different device structures were fabricated using metal/MoS2 contact - one with $\mathrm{Pd} / \mathrm{MoS}_{2}$ Schottky junction and the other with $\mathrm{Pd} / \mathrm{MoS}_{2} / \mathrm{Si}$ hetero-junction [40].

\section{The Edge CONTACTS}

Device and circuit fabrication technology employing very high charge carrier mobility in 2D-electron/hole gases (2DEGs/2DHGs), realized in bulk semiconductor based heterojunctions has already been put to use in recent past in producing high electron mobility transistors (HEMTs) for commercial applications in connection with micro and millimeter wave frequency devices extensively. Here, a thin sheet of 2DEG/2DHG are sandwiched between two higher band gap materials on either side constraining the charge carriers longitudinally but allowing them to move in transverse plane without any lattice scattering. Ohmic contacts to these 2DEG/2DHG sheets were invariably made through their edges [1], [41] - [43]. It is interesting to note that a similar scheme could possibly be used for making contacts to 2D-materials as reported recently [44].

Making ohmic contacts to a 2D semiconductor, in general, has thus two options namely - one is to deposit metal onto the extended surface and other onto the edges according to HEMT metallization scheme.

In case of metal deposition on surface, particularly the nonavailability of surface dangling bonds to establish chemical bonding through orbital hybridization results, in general, into large contact resistance across the planar metal and graphene monolayer interface [45] - [52]. For exploring edge contact metallization, the graphene sheet must be encapsulated in the form of h-BN/graphene/h-BN heterostructures (BN-G-BN) assembled for providing access to the graphene edges during metallization. Using conventional lithography to pattern and expose graphene surface is not favored in practice as the resins used are difficult to remove, besides their presence degrades the electrical contact, channel mobility, adding contaminations across the interface, causing bubbles and wrinkles that multiply with the addition of each successive layer, and limiting the typical device size to $~ 1 \mathrm{~mm}[53]-[59]$.

A novel technique described recently succeeded in establishing edge-contact to the graphene layer by encapsulating in form of a stack of h-BN and graphene in BN-G-BN configuration. A hard mask of hydrogensilsesquioxane (HSQ) resist applied onto the top BN surface of the stack allowed plasma etching to expose the edge. A composition of three metal layers (e.g. $1 \mathrm{~nm} \mathrm{Cr}, 15 \mathrm{~nm} \mathrm{Pd}$, and $60 \mathrm{~nm} \mathrm{Au}$ ) was e-beam deposited to establish electrical contact along the edge [44]. While characterizing this 1D contact, the carrier injection was noted to be limited to $1 \mathrm{D}$ atomic edge reducing the contact resistance to significantly low values $(\sim 100$ ohm.mm). Such fabricated graphene devices exhibited room-temperature mobility up to 140,000 $\mathrm{cm}^{2} / \mathrm{Vs}$ and sheet resistivity below $40 \mathrm{ohms} /$ square at $\mathrm{n}>$ $4.10^{12} / \mathrm{cm}^{2}$, approaching the theoretical limit due to acoustic phonon scattering. At temperatures below $40 \mathrm{~K}$, a ballistic transport was observed over $15 \mathrm{~mm}$ length scales [44], [47], [52], [60] - [62].

The STEM image did not show any metal diffusion into the graphene/BN interface, confirming the edge nature of the contact. The EELS map additionally indicated that the contact was based on $\mathrm{Cr}$ adhesion layer. The extensive measurements of contact resistances were found scaling inversely with the contact width, and is largely independent of temperature in contrast to the linear temperature scaling reported for surface contacts [44], [48].

\section{THE CURRENT STATUS}

Some recent experimental results reported in different R\&D laboratories are summarized here to highlight the situation regarding low contact fabrication of devices using 2D materials.

The electrical contacts to 2D-MLs would ideally need similarly thin electrode materials while maintaining low contact resistances. A scalable method was reported for fabricating edge-contacts to monolayer $\mathrm{MoS}_{2}$ and $\mathrm{WS}_{2}$ using graphene. The graphene and TMD layer were connected laterally with wafer-scale homogeneity, with no overlap or gap, and low average contact resistance of $30 \mathrm{k} \Omega \cdot \mu \mathrm{m}$ showing linear $I-V$-characteristics at room temperature was maintained down to liquid helium temperatures [63].

The intrinsic large band gap of $\mathrm{MoS}_{2}$ limits its application in IR detection beside its high mobility, low off-state current 
and high on/off ratio. A high-performance IR photo detector was, however, reported employing non-layers $\mathrm{PbS}$ and layered $\mathrm{MoS}_{2}$ nanostructures via van der Waals epitaxy. DFT calculations indicated that $\mathrm{PbS}$ nano plates are in contact with $\mathrm{MoS}_{2}$ edges through strong chemical hybridization, which offered enhanced response speed. The phototransistor exhibited a fast response $\left(\tau_{\text {rising }}, \tau_{\text {decay }}=\right.$ $7.8 \mathrm{~ms})$ as well as high photo responsivity $\sim 4.5 .10^{4} \mathrm{~A} / \mathrm{W}$, and $I_{\text {light }} / I_{\text {dark }} \sim 1.3 \times 10^{2}$ in the near-IR region at room temperature with $3.10^{13}$ Jones detectivity, which was even better than that of commercial $\mathrm{Si}$ and InGaAs photo detectors. Furthermore, by controlling the growth and micro fabrication patterning, periodic device arrays of $\mathrm{PbS}$ $\mathrm{MoS}_{2}$ were capable of IR detection achieved on $\mathrm{Si} / \mathrm{SiO}_{2}$ substrates [64].

Currently, few-layer graphene was demonstrated as a selfaligned edge-contacting scheme for layered material systems to take care of large contact resistance due to weak inter-layer coupling while circumventing the tunneling resistances of the weak coupling between layers [65].

A novel approach of forming low-resistance contacts to $\mathrm{MoS}_{2}$ transistors with the intrinsic performance of the channel was reported using a dry transfer technique and a metal-catalyzed graphene treatment process, nickel-etchedgraphene electrodes that were fabricated on $\mathrm{MoS}_{2}$ showing contact resistance $\sim 200 \Omega$. $\mu \mathrm{m}$. The fabrication of a clean graphene- $\mathrm{MoS}_{2}$ interface and a low resistance nickelgraphene interface is critical for experimental low contact resistance [66].

The scaling of $\mathrm{MoS}_{2}$ devices and contacts was examined with varying electrode metals and controlled deposition conditions, over a wide range of temperatures (80 to $500 \mathrm{~K}$ ), carrier densities $\left(10^{12}\right.$ to $\left.10^{13} / \mathrm{cm}^{2}\right)$, and contact dimensions (20 to $500 \mathrm{~nm}$ ). It was found that UHV deposited $\mathrm{Au}$ yielded three times lower $\mathrm{R}_{\mathrm{C}}$ than under normal conditions, reaching $740 \Omega \cdot \mu \mathrm{m}$ and specific contact resistivity $3 \times 10^{-}$ ${ }^{7} \Omega . \mathrm{cm}^{2}$, stable for over four months. The contact transfer length was $\sim 35 \mathrm{~nm}$ at $300 \mathrm{~K}$, as verified experimentally using devices with $20 \mathrm{~nm}$ contacts and $70 \mathrm{~nm}$ contact pitch corresponding to the $14 \mathrm{~nm}$ - technology node [67].

A new strategy of fabricating 2D/2D low-resistance ohmic contacts for a variety of transition metal dichalcogenides (TMDs) was reported using van der Waals assembly of substitutionally doped TMDs as drain/source contacts and TMDs with no intentional doping as channel materials. It was noted that few-layer $\mathrm{WSe}_{2}$ FETs with 2D/2D contacts exhibited low contact resistances of $\sim 0.3 \mathrm{k} \Omega \mu \mathrm{m}$, high on/off ratios up to $>10^{9}$, and high drive currents exceeding $320 \mu \mathrm{A} / \mu \mathrm{m}$. These favorable characteristics demonstrated a two-terminal field-effect hole mobility $\mu \approx 2.10^{2} \mathrm{~cm}^{2} / \mathrm{Vs} @$ room temperature, which increases to $>2.10^{3} \mathrm{~cm}^{2} / \mathrm{Vs} @$ cryogenic temperatures. A similar performance was also observed in $\mathrm{MoS}_{2}$ and $\mathrm{MoSe}_{2}$ FETs with 2D/2D drain and source contacts. The 2D/2D low-resistance ohmic contacts represented a new device paradigm that took care of a significant bottleneck in the performance of TMDs and a wide variety of other 2D materials as the channel materials in post silicon electronics [68].

A novel contact scheme utilizing cobalt (Co) with a monolayer of h-BN was noted to modify the work function of Co and provided for a tunneling barrier. A flat-band Schottky barrier of $16 \mathrm{meV}$ created a thin tunnel barrier upon doping the channels, and thus produced low contact resistance of $3 \mathrm{k} \Omega . \mu \mathrm{m}$ at a carrier density of $5.3 \times 10^{12} / \mathrm{cm}^{2}$ [69].

Despite the chemical inertness of h-BN, it is often used to encapsulate air-sensitive black phosphorus (BP). Its influence on SBH, doping, and contact resistance is not yet known. These effects were studied by fabricating h-BN encapsulated BP transistors with cobalt (Co) contacts. In sharp contrast to directly Co contacted p-type BP devices, strong n-type conduction was observed after inserting the h$\mathrm{BN}$ at the $\mathrm{Co} / \mathrm{BP}$ interface. First-principles calculations showed that this difference could be assigned to the larger interface dipoles at the $\mathrm{Co} / \mathrm{h}-\mathrm{BN}$ interface compared to the $\mathrm{Co} / \mathrm{BP}$ interface, which reduced the work function of the $\mathrm{Co} / \mathrm{h}-\mathrm{BN}$ contact. The $\mathrm{Co} / \mathrm{h}-\mathrm{BN}$ contacts thus exhibited low contact resistances $(\sim 4.5 \mathrm{k} \Omega)$ and were Schottky barrier-free [70].

$\mathrm{MoS}_{2}$ FETs were fabricated on PEN substrates with improved features using ultra short, pico-second pulsed laser anneal of $\mathrm{Ti}-\mathrm{Au}$ contacts without thermal damage. The reduced contact resistance obtained after such anneal provided significantly improved characteristics in terms increased mobility from 25 to $55 \mathrm{~cm}^{2} / \mathrm{Vs}, 3$ times increased output resistance, 6-fold self-gain and reduced sub-threshold swing. All these measured parameters confirmed reduction of SBH at $\mathrm{MoS}_{2}$-metal junction [71].

2D-metal carbides and nitrides also called MXenes are reported to produce Schottky-barrier-free metal contacts to 2D semiconductors, offering a solution to the contactresistance problem of $2 \mathrm{D}$ electronics. During ab-initio calculations, the surface chemistry was noted to strongly affect Fermi level of MXenes. For instance, O-terminations increased the work function compared to that of the bare surface; whereas $\mathrm{OH}$ always decreased it, and $\mathrm{F}$ exhibited either trend depending on the specific material. This phenomenon was assigned to the surface dipoles, which together with the weak Fermi level pinning, enabled Schottky-barrier-free hole (or electron) injection into 2D semiconductors through van der Waals junctions with some of the O-terminated (or all the OH-terminated) MXenes. This study could improve the understanding of the correlation between surface chemistry and electronic transport properties of 2D materials, which are found promising to improve the 2D-electronic devices [72].

MXenes represent an emerging family of 2D-materials that are applicable in termination-mediated surface functionalization, though understanding of the termination is rather limited as yet. Taking the example of $\mathrm{Ti}_{3} \mathrm{C}_{2} \mathrm{X}_{2}$ (where, $X=\mathrm{O}, \mathrm{F}, \mathrm{OH}$, and $\mathrm{H}$ ) MXenes, termination led stabilization was demonstrated in $\mathrm{Ti}_{3} \mathrm{C}_{2}$ monolayer matrix via nonbonding valence electron saturations of the surface $\mathrm{Ti}$ atom by the low-energy orbitals of the termination. Highly degenerate 3d-orbitals of surface Ti were found splitting in such a way that they exhibited pseudo gaps widths resulting in a stability order of $\mathrm{Ti}_{3} \mathrm{C}_{2} \mathrm{O}_{2}>\mathrm{Ti}_{3} \mathrm{C}_{2} \mathrm{~F}_{2}>\mathrm{Ti}_{3} \mathrm{C}_{2}(\mathrm{OH})_{2}>$ $\mathrm{Ti}_{3} \mathrm{C}_{2} \mathrm{H}_{2}>\mathrm{Ti}_{3} \mathrm{C}_{2}$, consistent with the theoretical and experimental results [73].

Arsenene (arsenic analogue of graphene) was examined in the context of its ML contact properties with different 
electrodes using simulations. Invariably Schottky barrier was formed with bulk metal contacts owing to the Fermi level pinning with electron SBH of $0.12,0.21,0.25,0.35$, and $0.50 \mathrm{eV}$ for $\mathrm{Sc}, \mathrm{Ti}, \mathrm{Ag}, \mathrm{Cu}$, and $\mathrm{Au}$ contacts and hole $\mathrm{SBH}$ of 0.75 and $0.78 \mathrm{eV}$ for $\mathrm{Pd}$ and $\mathrm{Pt}$ contacts, respectively. However, by contacting with $2 \mathrm{D}$-graphene, the Fermi level pinning effect was found reduced due to the suppression of metal-induced gap states. A barrier free hole injection was thus realized in arsenene ML with GraphenePt hybrid electrode resulting in a high performance device [74].

2D-perovskite $\left(\mathrm{C}_{4} \mathrm{H}_{9} \mathrm{NH}_{3}\right)_{2} \mathrm{PbX}_{4}(\mathrm{Cl}, \mathrm{Br}, \mathrm{I})$ channels were prepared and contacted with graphene and other $2 \mathrm{D}$ metallic sheets to form vdW hetero-structures for FET devices. Using modern simulations, the intrinsic properties of the 2D-perovskites were noted to remain intact in the heterojunction in contrast to the conventional contact with metal surfaces. The 2D-perovskites formed a p-type Schottky barrier contact with graphene with tunneling barrier and a negative band bending at the lateral interface. SB could thus be turned from p-type to n-type by doping graphene with nitrogen atoms. This presented Ohmic contacts by doping graphene with boron atoms or replacing graphene with other high-work-function 2D-metal sheets like $Z T-\mathrm{MoS}_{2}, Z T$ $\mathrm{MoSe}_{2}$ and $H-\mathrm{NbS}_{2}$ [75].

In all transition-metal dichalcogenides monolayers (MLs), the metal centers possess a spin, and their ground-state phases are either metallic or semiconducting with indirect band gaps. Using first-principle calculations, the Haeckelite polytypes $1 \mathrm{~S}-\mathrm{NbX}_{2}(\mathrm{X}=\mathrm{S}, \mathrm{Se}, \mathrm{Te})$ were observed with diamagnetic direct-band-gaps even though the $\mathrm{Nb}$ atoms were in the $4^{+}$oxidation states. In contrast, $1 \mathrm{~S}-\mathrm{VX}_{2} \mathrm{MLs}$ were anti-ferro-magnetically coupled indirect-band-gap semiconductors. The $1 \mathrm{~S}$ phases were noted to be thermodynamically stable but of slightly higher energy than their $1 \mathrm{H}$ and $1 \mathrm{~T} \mathrm{ML}$ counterparts. $1 \mathrm{~S}-\mathrm{NbX}_{2} \mathrm{MLs}$ were found useful in optoelectronic applications owing to their small band gaps (between 0.5 and $1 \mathrm{eV}$ ). Moreover, $1 \mathrm{~S}$ $\mathrm{NbS}_{2}$ showed a particularly high hole-mobility of $2.68 .10^{3}$ $\mathrm{cm}^{2} / \mathrm{Vs}$, which was significantly higher than that of $\mathrm{MoS}_{2}$ and comparable to that of $\mathrm{WSe}_{2}$ [76].

Phosphorene is currently being examined as an alternative channel material for electronic and optoelectronic devices. Compared with ML phosphorene, FL-phosphorene is easier to prepare, and is more stable in experiments forming a smaller SBH at the phosphorene-metal interface. Using quantum simulations, the interface properties of three-layer (3L) phosphorene FETs were studied by contacting with metals like Al, Ag, Au, Cu, Ti, Cr, Ni, and Pd. The SBHs obtained in the vertical direction from projecting the band structures of the $3 \mathrm{~L}$ phosphorene-metal systems to the left bilayer (2L)-phosphorenes were found comparable with those in the lateral direction from the simulations. It was further observed in case of $3 \mathrm{~L}$ phosphorene FETs that it formed n-type Schottky contacts with electron SBHs of 0.16 and $0.28 \mathrm{eV}$ in the lateral direction, when $\mathrm{Ag}$ and $\mathrm{Cu}$ were used as electrodes, respectively, and p-type Schottky contacts with hole SBHs of 0.05, 0.11, 0.20, 0.30, 0.30, and $0.31 \mathrm{eV}$ in the lateral direction when $\mathrm{Cr}, \mathrm{Pd}, \mathrm{Ni}, \mathrm{Ti}, \mathrm{Al}$, and $\mathrm{Au}$ were used as electrodes, respectively. These simulation resuls were found in agreement with the available experimental results [77].

The work functions of 2D-metal carbides and nitrides were studied by considering the induced surface dipoles and Fermi level shifts. A correlation could thus be established between the work function and the adsorbate's 2p-band centres. Taking the example of $\mathrm{OH}$-terminated MXenes a relation between the $2 p$ band centres and workfunctions was demonstrated. Under these consideratins, a limit on the lowest possible work function of $\sim 1.2 \mathrm{eV}$ was shown. The optimum value of the $2 \mathrm{p}$-band centres at which this low work function occured was determined to be $\sim-5.45 \mathrm{eV}$ relative to the Fermi level [78].

Band gap modification in case of $\mathrm{Sc}_{2} \mathrm{CF}_{2}$ MXene MLs resulting from substitution of $\mathrm{C}$ atoms by $\mathrm{Si}, \mathrm{Ge}, \mathrm{Sn}, \mathrm{F}, \mathrm{S}$, $\mathrm{N}, \mathrm{B}$, and $\mathrm{B}+\mathrm{N}$ was studied using DFT calculations. During this study it was noted that $\mathrm{Si}, \mathrm{Ge}, \mathrm{Sn}, \mathrm{B}$, and $\mathrm{B}+\mathrm{N}$ doped $\mathrm{Sc}_{2} \mathrm{CF}_{2}$ MLs remain semiconducting (with band gap from 0.55 to $0.24 \mathrm{eV}$ ). Semiconductor to metal transition was also observed in F, S, and $\mathrm{N}$ doped MLs and in $\mathrm{C}$ mono vacancy. In the B doped MLs, spin polarization due to the half filling of a defect band was observed. The substitutional doping of the MXene monolayer by $\mathrm{F}, \mathrm{N}, \mathrm{S}$, and $\mathrm{B}+\mathrm{N}$ were found favorable, and energy penalties for $\mathrm{Si}, \mathrm{Ge}$, and $\mathrm{Sn}$ were not formidable. On the other hand, the substitution in the bulk $\mathrm{Sc}_{2} \mathrm{AlC}$ MAX phase, the precursor of the $\mathrm{Sc}_{2} \mathrm{CF}_{2} \mathrm{ML}$, was exothermic for all substituents. C substitution in the $\mathrm{Sc}_{2} \mathrm{CF}_{2} \mathrm{MXene}$ MLs was found useful in adjusting the electronic properties [79].

Selective crystal polymorphism of one ML TMDC as metallic or semiconducting phase was explored to integrate the resultant devices in electronic circuitry. Developing an appropriate growth strategy for such polymorphs in the vapor phase is known as a critical step in this endeavor of integrating metallic $\left(1 \mathrm{~T}^{\prime}\right)$ and semiconducting $(2 \mathrm{H})$ $\mathrm{MoTe}_{2}$ crystals by heteroepitaxy. Such a polymorphic coplanar contact was atomically coherent, and its potential barrier was spatially confined over a length of only a few $\mathrm{nm}$, with a lowest contact barrier height of $\sim 25 \mathrm{meV}$ [80].

The contact resistances of "end-contacted" metal electrode-graphene, and CNT-interfaces were studied theoretically with $\mathrm{Ti}, \mathrm{Pd}, \mathrm{Pt}, \mathrm{Cu}$, and $\mathrm{Au}$. The contact resistances (normalized to surface $\mathrm{C}$ atoms) were 107, 142, 149, 253, and $187 \mathrm{k} \Omega$ for $\mathrm{Ti}, \mathrm{Pd}, \mathrm{Pt}, \mathrm{Cu}$, and $\mathrm{Au}$, respectively. This was compared with the contact resistance (per C) for "side-contacted" metal-graphene or metal-CNT interfaces with contact resistance of 8.6, 34.7, and $630 \mathrm{M} \Omega$ for $\mathrm{Pd}, \mathrm{Pt}$, and $\mathrm{Cu}$, respectively. These results were noted to be in good agreement with available experimental results, $40.5 \mathrm{M} \Omega$ for Pt. This study suggested developing a technology to achieve "end-contacted" configurations [81].

Comparison of edge versus surface-contacted ferromagnetic metal-graphene interfaces (i.e., $\mathrm{Ni}$ and $\mathrm{Co}-$ graphene interfaces) was made in both ML/few-layer structures. Qualitative agreement was obtained between theory and experiment. The larger contact resistance in surface contacts resulted from spin filtering at the interface, in contrast to the edge-contacted cases where both spins were transmitted. Temperature-dependent resistance measurements beyond the Curie temperature $T_{C}$ showed that 
the spin degree of freedom was important for the measured contact resistance [82].

Forming contacts to graphene MLs from the edges instead of those from the top were found superior in realizing low ohmic contacts. Fabrication of edge contacts by means of optical lithography and CMOS compatible metals (i.e. Nickel and Aluminum) was reported in large area CVDgrown graphene MLs. A very low width specific contact resistance down to $130 \Omega$. $\mu \mathrm{m}$ was extracted from the characterizations. The contact resistance was found stable during anneal up to $150^{\circ} \mathrm{C}$. Using this type of edge contact scheme MOSFETs were fabricated on CVD graphene with a high trans conductance of 0.63 mS/ $\mu \mathrm{m} @ 1 \mathrm{~V}$ [83].

Challenges faced in the integration of 2D/3D-systems were studied in detail recently. The main observations pointed towards improving contact resistivity of metal on graphene and related 2D-materials, while Schottky junctions comprising of metal/2D-semiconductor or graphene/3Dsemiconductor needed better physical models of junction behavior. Recent developments in preparing 2D/3D semiconductor junctions and their future applications were reviewed briefly [84].

Improved interfaces from alloyed 2D-metalsemiconductor hetero-junction were explored in $2 \mathrm{D}-\mathrm{WSe}_{2}-$ transistors with mixed transition layers containing atomically sharp interfaces across $\mathrm{M}-\mathrm{vdW}$ comprising of $\mathrm{NbSe}_{2} / \mathrm{W}_{\mathrm{x}} \mathrm{Nb}_{1-\mathrm{x}} \mathrm{Se}_{2} / \mathrm{WSe}_{2}$. Such alloyed contact devices exhibited about 80 times longer carrier lifetimes than that of $\mathrm{Pd} / \mathrm{WSe}_{2}$ junctions. Such lifetime improvements were attributed to the defect reduction and Schottky barrier lowering. Formation of a controllable mixed-composition alloyed layer on the 2D-channel is a breakthrough approach for maximizing the stability and reliability of 2D nanomaterials applications [85].

\section{DisCUSSION AND CONCLUSIONS}

Passing through the development of $3 \mathrm{D}$ to $2 \mathrm{D}$ thin film FETs a number of problems associated with conventional FETs are overcome by incorporating several types of new changes. For instance, 2D-FETs are free from short-channel effects because all the electrons are confined in atomically thin natural channels that are dangling bonds free at the surface. Moreover, single and few-layer form of layered 2Dmaterials have a wide range of tunable electrical and optical properties, atomic-scale thickness, mechanical flexibility and large band gaps $(1 \sim 2 \mathrm{eV})$. However, the major problem with $2 \mathrm{D}$ FETs is the large contact resistance at the interface between the 2D semiconductor and any bulk metal. To address to this problem a new technique of producing 2D transistors was reported with semiconductor and metal made of the same chemical compound such as molybdenum telluride $\left(\mathrm{MoTe}_{2}\right)$. Being polymorphic, it could be used both as metal and semiconductor. Contact resistance at the interface between the semiconductor and metallic $\mathrm{MoTe}_{2}$ was shown to be very low. Barrier height was reduced from 150 to $22 \mathrm{meV}$ [86], [87].

Taking a hint from the observation mentioned in the text, it seems graphene monolayer is necessary to insert between 2D-semiconductor and metal for which a detailed theoretical modeling followed by appropriate technology seems to be necessary to develop. For minimizing the disturbances caused to the monolayer graphene from the presence of metal layer may be possibly designed using multiple layers of metal using atomic layer epitaxy (ALE). Similarly, inserting something similar to h-BN monolayer should control the influence of multiple layers of $2 \mathrm{D}$-semiconductor lying below. Besides, some species like $\mathrm{O}_{2}$ or other may be attached to the interface to provide better stability and control in device fabrication.

Full potentials of 2D-materials in fabricating individual and integrated devices will be possible after realizing reliable and reproducible ohmic and Schottky contacts with precisely controlled interfaces. In absence of sufficient experimental methods explored so far most of the possibilities of improving the contact properties are first studied theoretically using structural as well as charge carrier transport properties. Theoretical and experimental observations made so far give a strong evidence of realizing improved 2D-semiconductor based devices using graphene monolayer sheet in combination with a set of metal thin films in near future. Exploring planar and edge-contacts with overhanging metal layer might be a better choice than any one technique alone. Use of ionic liquid and h-BN offers another possibility as mentioned earlier in the text. It could further be noted that laser annealed metal combination have also produced reasonable good ohmic contacts, which were found very much similar to $3 \mathrm{D}$-ohmic contacts.

The current status of mixed observations will perhaps improve with more experience in establishing vdW epitaxy where atomically sharp interfaces will be ultimately possible to fabricate with not much constraints compared to conventional homo-epitaxy.

\section{ACKNOWLEDGMENT}

The authors express their gratitude to the research colleagues in YMCA University, for very active interactions and involved discussions. Prof. Dinesh Kumar, VC, YMCA University of Science \& Technology, deserves special thanks for his kind encouragements and support throughout.

\section{REFERENCES}

[1] S. Ahmad, Micro and Millimeter Wave Semiconductor Device Technology. Tata McGraw Hill, Delhi, India, 1998.

[2] A. Allain, J. Kang, K. Banerjee, and A. Kis, "Electrical contacts to two-dimensional semiconductors," Nature Materials, vol. 14, pp. 1195-1205, 2015.

[3] O. Lopez-Sanchez, D. Lembke, M. Kayci, A. Radenovic, and A. Kis, "Ultrasensitive photo detectors based on monolayer MoS2," Nature Nanotech., vol. 8, pp. 497-501, 2013.

[4] D. Krasnozhon, D. Lembke, C. Nyffeler, Y. Leblebici, and A. Kis, "MoS2 transistors operating at gigahertz frequencies," Nano Lett., vol. 14, pp. 5905-11, 2014.

[5] S. Das, H. Y. Chen, A. V. Penumatcha, and J. Appenzeller, (2013), "High performance multilayer MoS2 transistors with scandium contacts," Nano Lett., vol. 13, pp. 100-105, 2013.

[6] H. Liu, A. T. Neal, and P. D. Ye, "Channel length scaling of MoS2 MOSFETs," ACS Nano., vol. 6, pp. 8563-9, 2012.

[7] H. Liu, M. Si, S. Najmaei, A. T. Neal, Y. Du, P. M. Ajayan, J. Lou, and P. D. Ye, "Statistical study of deep submicron dual-gated fieldeffect transistors on monolayer chemical vapor deposition molybdenum disulfide films," Nano Lett., vol. 13, pp. 2640-6, 2013.

[8] R. Landauer, "Spatial variation of currents and fields due to localized scatterers in metallic conduction," IBM J. Res. Dev. vol.1, pp. 223231, 1957.

[9] Y. V. Sharvin, "A possible method for studying Fermi surfaces," Sov. Phys. JETP, vol. 21, pp. 655-6, 1965.

[10] D. Jena, K. Banerjee, and G. H. Xing, "2D crystal semiconductors: Intimate contacts," Nature Mater., vol. 13, pp. 1076-8, 2014. 
[11] G. T. Wright, "Small-signal theory of the transistor transit-time oscillator/translator/," Solid-State Electronics, vol. 22(4), pp. 399403, 1979.

[12] S. M. Sze, Physics of Semiconductor Devices, Wiley-Inter science, 1969.

[13] J. Bardeen, "Surface States and Rectification at a Metal SemiConductor Contact," Phys. Rev. vol. 71, 717, 1947.

[14] Y. Xu, C. Cheng, S. Du, J. Yang, Bin Yu, J. Luo, W. Yin, E. Li, S. Dong, P. Ye, and X. Duan, "Contacts between Two and ThreeDimensional Materials: Ohmic, Schottky, and p-n Hetero junctions,' ACS Nano, vol. 10 (5), pp. 4895-919, 2016.

[15] S. Das, H.-Y. Chen, A. V. Penumatcha, and J. Appenzeller, "HighPerformance Multilayer MoS2 Transistors with Scandium Contacts," Nano Lett., 13 (1), pp. 100-105, 2013.

[16] Y. Zhao, X. Xiao, Y. Huo, Y. Wang, T. Zhang, K. Jiang, J. Wang, S. Fan, and Q. Li, "Influence of Asymmetric Contact Form on Contact Resistance and Schottky Barrier, and Corresponding Applications of Diode," ACS Appl. Mater. Interfaces, vol. 9 (22), pp.18945-55, 2017.

[17] K. S. Kim, K. H. Kim, Y. Nam, J. Jeon, S. Yim, E. Singh, J. Y. Lee, S. J. Lee, Y. S. Jung, G. Y. Yeom, and D. W. Kim, “Atomic layer etching mechanism of MoS2 for nano devices," ACS Appl. Mater. Interfaces, vol. 9 (13), pp. 11967-76, 2017.

[18] S. Roy, G. P. Neupane, K. P. Dhakal, J. Lee, S. J. Yun, G. H. Han, and J. Kim, "Observation of Charge transfer in heterostructures composed of MoSe2 quantum dots and a monolayer of MoS2 or WSe2," J. Phys. Chem. C, vol. 121 (3), pp. 1997-2004, 2017.

[19] M.-H. Doan, Y. Jin, S. Adhikari, S. Lee, J. Zhao, S. C. Lim, and Y. H. Lee, "Charge transport in MoS2/WSe2 van der Waals hetero-structure with tunable inversion layer," ACS Nano, vol. 11 (4), pp. 3832-40, 2017.

[20] J. S. Ross, P. Rivera, J. Schaibley, E. Lee-Wong, H. Yu, T. Taniguchi, K. Watanabe, J. Yan, D. Mandrus, D. Cobden, W. Yao, and X. Xu, (2017), Interlayer exciton optoelectronics in a 2D hetero structure $\mathrm{p}-\mathrm{n}$ Junction," Nano Lett., vol. 17 (2), pp. 638-43, 2017.

[21] K. Xu, D. Chen, F. Yang, Z. Wang, L. Yin, F. Wang, R. Cheng, K. Liu, J. Xiong, Q. Liu, and J. He, "Sub-10nm nano pattern architecture for 2D material field-effect transistors," Nano Lett., vol. 17 (2), pp. 1065-70, 2017.

[22] C. Kim, I. Moon, D. Lee, M. S. Choi, F. Ahmed, S. Nam, Y. Cho, H.J. Shin, S. Park, and W. J. Yoo, "Fermi level pinning at electrica metal contacts of monolayer Molybdenum Dichalcogenides," ACS Nano, vol.11 (2), pp.1588-96, 2017.

[23] J. Guan, H.-J. Chuang, Z. Zhou, and D. Tománek, “Optimizing charge injection across transition metal di-chalcogenide heterojunctions: Theory and Experiment," ACS Nano, vol. 11 (4), pp. 3904-10, 2017.

[24] C. Zheng, Q. Zhang, B. Weber, H. Ilatikhameneh, F. Chen, H. Sahasrabudhe, R. Rahman, S. Li, Z. Chen, J. Hellerstedt, Y. Zhang, W. H. Duan, Q. Bao, and M. S. Fuhrer, "Direct observation of 2D electrostatics and ohmic contacts in template-grown graphene/WS2 heterostructures," ACS Nano, vol. 11 (3), pp. 2785-93, 2017.

[25] D. Stradi, N. R. Papior, O. Hansen, and M. Brandbyge, "Field Effect in graphene-based van der Waals heterostructures: Stacking sequence matters," Nano Lett., vol. 17 (4), pp. 2660-6, 2017.

[26] R. Li, L. Zhang, L. Shi, and P. Wang, "MXene Ti3C2: An effective 2D light-to-heat conversion material," ACS Nano, vol. 11 (4), pp. 3752-9, 2017

[27] H. Lin, X. Wang, L. Yu, Y. Chen, and J. Shi, "Two-dimensional ultrathin MXene ceramic nanosheets for photo-thermal conversion," Nano Lett., vol. 17 (1), pp. 384-91, 2017.

[28] L. Huang, B. Li, M. Zhong, Z. Wei, and J. Li, "Tunable Schottky barrier at MoSe2/Metal interfaces with a buffer layer," J. Phys. Chem. C, vol. 121 (17), pp. 9305-11, 2017.

[29] Y. Yoon, K. Ganapathi, and S. Salahuddin, "How good can monolayer MoS2 transistors be?" Nano Lett., vol. 11 (9), pp. 3768 73,2011 .

[30] H. Wang, L. Yu, Y.-H. Lee, Y. Shi, A. Hsu, M. L. Chin, L.-J. Li, M. Dubey, J. Kong, and T. Palacios, "Integrated circuits based on bilayer MoS2 transistors," Nano Lett., vol. 12 (9), pp. 4674-80, 2012.

[31] S. Sattar and U. Schwingenschlögl, "Electronic properties of graphene-PtSe2 contacts,” ACS Appl. Mater. Interfaces, vol. 9 (18), pp. 15809-13, 2017.

[32] H. G. Kim, and H.-B.-R. Lee, "Atomic layer deposition on 2D materials," Chem. Mater., vol. 29(9), pp. 3809-26, 2017.

[33] A. Nourbakhsh, A. Zubair, R. N. Sajjad, A. Tavakkoli K. G. W. Chen, S. Fang, X. Ling, J. Kong, M. S. Dresselhaus, E. Kaxiras, K. K. Berggren, D. Antoniadis, and T. Palacios, "MoS2 field-effect transistor with sub-10nm channel length," Nano Lett. vol. 16 (12), pp. 7798-7806, 2016.
[34] W. S. Leong, X. Luo, Y. Li, K. H. Khoo, S. Y. Quek, and J. T. L. Thong, "Low resistance metal contacts to MoS2 devices with nickeletched-graphene electrodes," ACS Nano, vol. 9 (1), pp. 869-77, 2015.

[35] H.-J. Chuang, B. Chamlagain, M. Koehler, M. M. Perera, J. Yan, D. Mandrus, D. Tománek, and Z. Zhou, "Low-resistance 2D/2D ohmic contacts: A universal approach to high-performance WSe2, MoS2, and MoSe2 transistors," Nano Lett., vol. 16 (3), pp. 1896-1902, 2016.

[36] Y. Kim, A. R. Kim, J. H. Yang, K. E. Chang, J.-D. Kwon, S. Y. Choi, J. Park, K. E. Lee, D.-H. Kim, S. M. Choi, K. H. Lee, B. H. Lee, M G. Hahm, and B. Cho, "Alloyed 2D metal-semiconductor heterojunctions: Origin of interface states reduction and Schottky barrier Lowering," Nano Lett., vol. 16 (9), pp. 5928-33, 2016.

[37] Y. Sata, R. Moriya, S. Masubuchi, K. Watanabe, T. Taniguchi, and T. Machida, "N and P-type carrier injections into WSe2 with van der Waals contacts of two-dimensional materials,” Jap. J. App. Phys., vol. 56, 04CK09, 2017.

[38] D. Kufer, and G. Konstantatos, "Photo-FETs: Phototransistors enabled by 2D and 0D Nanomaterials," ACS Photonics, vol. 3 (12) pp. 2197-2210, 2016.

[39] H. Zhong, R. Quhe, Y. Wang, Z. Ni, M. Ye, Z. Song, Y. Pan, J. Yang, L. Yang, M. Lei, J. Shi, and J. Lu, "Interfacial properties of monolayer and bilayer MoS2 contacts with metals: Beyond the energy band calculations," Scientific Rep. 6:21786, 2016. DOI: 10.1038/ srep21786

[40] C. P. Y. Wong, C. Troadec, K. E. J. Goh, and A. T. S. Wee, “A study of the metal/2D semiconductor contacts," 2017. Text @ www.physics.nus.edu.sg/ surface/Posters 20Wong_Poster\%20-\%20ISRF\%20 2015.pdf

[41] A. Bin Khudhayr 2017 Text http://userweb.eng.gla.ac.uk/MScPosters2014-15/ Engineering\%20and\%20Management/EEE\%20 and $\% 20$ Management/Developing\%20of\%20Lowresistance $\% 200 \mathrm{Omi}$ c\%20Contact\%20on\%20GaN \% 20 HEMTs.pdf

[42] D. Taneja, F. Sfigakis, A. F. Croxall, K. Das Gupta, V. Narayan, J. Waldie, I. Farrer, and D. A. Ritchie, "N-type ohmic contacts to undoped GaAs/AlGaAs quantum wells using only front-sided processing: application to ambipolar FETs," Semicond. Sci. Technol., vol. 31, 065013 (7pp), 2016.

[43] P. Parikh, Y. Wu, and L. Shen, "Commercialization of high 600V GaN-on-silicon power HEMTs and diodes. IEEE Energytech., pp. 21 23, May 2013; DOI: 10.1109/EnergyTech. 2013.6645300

[44] L. Wang, I. Meric, P. Y. Huang, Q. Gao, Y. Gao, H. Tran, T. Taniguchi, K. Watanabe, L. M. Campos, D. A. Muller, J. Guo, P. Kim, J. Hone, K. L. Shepard, and C. R. Dean, "One-dimensional electrical contact to a two-dimensional material," Science, vol. 342, pp. 614-7, 2013.

[45] G. Giovannetti, P. A. Khomyakov, G. Brocks, V. M. Karpan, J. van den Brink, and P. J. Kelly, "Doping graphene with metal contacts," Phys. Rev. Lett., vol. 101, 026803, 2008.

[46] Q. Tang, C. X. Zhang, C. He, C. Tang, and J. Zhong, "Charge transport properties of graphene: Effects of $\mathrm{Cu}$-based gate electrode," J. Appl. Phys., vol.108, 123711, 2010.

[47] F. Léonard, and A. A. Talin, "Electrical contacts to one- and twodimensional nanomaterials," Nat. Nanotechnol., vol. 6, pp.773-83, 2011.

[48] F. Xia, V. Perebeinos, Y. M. Lin, Y. Wu, and P. Avouris, "The origins and limits of metal-graphene junction resistance. Nat. Nanotechnol. 6, 179-84, 2011

[49] M. S. Choi, S. H. Lee, and W. J. Yoo, "Plasma treatments to improve metal contacts in graphene field effect transistor," J. Appl. Phys., vol. 110, 073305, 2011.

[50] D. Berdebes, T. Low, Y. Sui, J. Appenzeller, and M. S. Lundstrom, "Substrate gating of contact resistance in graphene transistors," IEEE Trans. Electron. Dev., vol. 58, pp. 3925-32, 2011.

[51] J. S. Moon, M. Antcliffe, H. C. Seo, D. Curtis, S. Lin, A. Schmitz, I Milosavljevic, A. A. Kiselev, R. S. Ross, D. K. Gaskill, P. M. Campbell, R. C. Fitch, K.-M. Lee, and P. Asbeck, "Ultra-low resistance ohmic contacts in graphene field effect transistors," Appl Phys. Lett. vol.100, 203512, 2012.

[52] J. T. Smith, A. D. Franklin, D. B. Farmer, and C. D. Dimitrakopoulos, "Reducing contact resistance in graphene devices through contact area patterning," ACS Nano vol. 7, pp. 3661-7, 2013.

[53] A. K. Geim, and I. V. Grigorieva, "van der Waals heterostructures," Nature vol. 499, pp. 419-25, 2013.

[54] A. S. Mayorov, R. V. Gorbachev, S. V. Morozov, L. Britnell, R. Jalil, L. A. Ponomarenko, P. Blake, K. S. Novoselov, K. Watanabe, T. Taniguchi, and A. K. Geim, "Micrometer-scale ballistic transport in encapsulated graphene at room temperature," Nano Lett., vol. 11, pp 2396-9, 2011. 
[55] M. Ishigami, J. H. Chen, W. G. Cullen, M. S. Fuhrer, and E. D. Williams, "Atomic structure of graphene on SiO2," Nano Lett., vol.7, pp. 1643-1648, 2007.

[56] J. A. Robinson, M. LaBella, M. Zhu, M. Hollander, R. Kasarda, Z. Hughes, K. Trumbull, R. Cavalero, and D. Snyder, "Contacting graphene," Appl. Phys. Lett., vol. 98, 053103, 2011.

[57] J. Yamaguchi, K. Hayashi, S. Sato, and N. Yokoyama, "Passivating chemical vapor deposited graphene with metal oxides for transfer and transistor fabrication processes," Appl. Phys. Lett., vol. 102, 143505, 2013.

[58] N. Lindvall, A. Kalabukhov, and A. Yurgens, "Erratum: "Cleaning graphene using atomic force microscope," [J. Appl. Phys., vol. 111, 064904, 2012], J. Appl. Phys. vol. 111, 064904, 2012.

[59] S. J. Haigh, A. Gholinia, R. Jalil, S. Romani, L. Britnell, D. C. Elias, K. S. Novoselov, L. A. Ponomarenko, A. K. Geim, and R. Gorbachev, "Cross-sectional imaging of individual layers and buried interfaces of graphene-based heterostructures and superlattices," Nat. Mater., vol. 11, pp. 764-7, 2012.

[60] Y. Matsuda, W.-Q. Deng, And W. A. Goddard Iii, "Contact Resistance for "End-Contacted" Metal-Graphene and Metal-Nanotube Interfaces from Quantum Mechanics," J. Phys. Chem. C, Vol. 114, Pp. 17845-50, 2010.

[61] K. Cho, "First-Principles and Quantum Transport Studies of MetalGraphene End Contacts," Mrs Proc., Vol., 1259, Pp. S14-S35, 2010.

[62] Y. Wu, Y. Wang, J. Wang, M. Zhou, A. Zhang, C. Zhang, Y. Yang, Y. Hua, and B. Xu, "Electrical transport across metal-twodimensional carbon junctions: edge versus side contacts," AIP Adv., vol. 2(1), 012132, 2012.

[63] M. H. D. Guimarães, H. Gao, Y. Han, K. Kang, S. Xie, C.-J. Kim, D A. Muller, D. C. Ralph, J. Park, "Atomically Thin Ohmic Edge Contacts Between Two-Dimensional Materials," Acs Nano., Vol. 10 (6), Pp. 6392-9, 2016.

[64] Y. Wen, L. Yin, P. He, Z. Wang, X. K. Zhang, Q. Wang, T. A. Shifa K. Xu, F. Wang, X. Zhan, F. Wang, C. Jiang, And J. He, "Integrated High-Performance Infrared Phototransistor Arrays Composed of NonLayered Pbs-Mos2 Heterostructures with Edge Contacts," Nano Lett., Vol. 16 (10), Pp. 6437-6444, 2016.

[65] T. Chu and Z. Chen, "Understanding The Electrical Impact of Edge Contacts in Few-Layer Graphene," Acs Nano, Vol. 8 (4), Pp. 3584-9, 2014.

[66] W. S. Leong, X. Luo, Y. Li, K. H. Khoo, S. Y. Quek, And J. T. L. Thong, "Low-Resistance Metal Contacts to Mos2 Devices with Nickel-Etched-Graphene Electrodes," Acs Nano, Vol. 9 (1), Pp. 869 $877,2015$.

[67] C. D. English, G. Shine, V. E. Dorgan, K. C. Saraswat, And E. Pop, "Improved Contacts to Mos2 Transistors by Ultra-High Vacuum Metal Deposition," Nano Lett., Vol. 16 (6), Pp. 3824-30, 2016.

[68] H. -J. Chuang, B. Chamlagain, M. Koehler, M. M. Perera, J. Yan, D. Mandrus, D. Tománek, and Z. Zhou, "Low-resistance 2D/2D ohmic contacts: A universal approach to high-performance WSe2, MoS2 and MoSe2 transistors," Nano Lett., vol. 16 (3), pp. 1896-1902, 2016.

[69] X. Cui, E.-M. Shih, L. A. Jauregui, S. H. Chae, Y. D. Kim, B. Li, D. Seo, K. Pistunova, J. Yin, J.-H. Park, H.-J. Choi, Y. H. Lee, K. Watanabe, T. Taniguchi, P. Kim, C. R. Dean, and J. C. Hone, "Lowtemperature ohmic contact to monolayer MoS2 by van der Waals bonded Co/h-BN electrodes," Nano Lett., vol. 17 (8), pp. 4781-6, 2017.

[70] A. Avsar, J. Y. Tan, X. Luo, K. H. Khoo, Y. Yeo, K. Watanabe, T. Taniguchi, S. Y. Quek, and B. Özyilmaz, "van der Waals bonded Co/h-BN contacts to ultrathin black phosphorus devices," Nano Lett., vol. 17 (9), pp. 5361-7, 2017.

[71] H.-J. Kwon, W. Choi, D. Lee, Y. Lee, J. Kwon, B. Yoo, C. P. Grigoropoulos, and S. Kim, "Selective and localized laser-anneal effect for high-performance flexible multilayer MoS2 thin-film transistors," Nano Res., vol. 7, 1137, 2014.
[72] Y. Liu, H. Xiao, and W. A. Goddard, "Schottky-barrier-free contacts with two-dimensional semiconductors by surface-engineered MXenes,” J. Am. Chem. Soc., vol. 138 (49), pp. 15853-6, 2016.

[73] T. Hu, Z. Li, M. Hu, J. Wang, Q. Hu, Q. Li, and X. Wang, "Chemica origin of termination-functionalized MXenes: Ti3C2T2 as a case study," J. Phys. Chem. C, vol. 121 (35), pp. 19254-61, 2017.

[74] Y. Wang, M. Ye, M. Weng, J. Li, X. Zhang, H. Zhang, Y. Guo, Y. Pan, L. Xiao, J. Liu, F. Pan, and J. Lu, "Electrical contacts in monolayer arsenene devices," ACS Appl. Mater. Interfaces, vol. 9 (34), pp. 29273-84, 2017.

[75] Y. Guo, W. A. Saidi, and Q. Wang, "2D halide perovskite-based van der Waals heterostructures: contact evaluation and performance modulation," 2D Materials, vol. 4(3), 035009, 2017.

[76] Y. Ma, A. Kuc, Y. Jing, P. Philipsen, and T. Heine, "Twodimensional haeckelite $\mathrm{NbS2}$ : A diamagnetic high-mobility semiconductor with $\mathrm{Nb} 4+$ ions," Angew. Chem. Int. Ed., vol. 56 , 10214, 2017

[77] X. Zhang, Y. Pan, M. Ye, R. Quhe, Y. Wang, Y. Guo, H. Zhang, Y. Dan, Z. Song, J. Li, J. Yang, W. Guo, and J. Lu, "Three-layer phosphorene-metal interfaces," Nano Res., 2017; https://doi.org/10. 1007/s12274-017-1680-6

[78] H. A. Tahini, X. Tan, and S. C. Smith, "The origin of low work functions in OH-terminated MXenes," Nanoscale, vol. 9, pp. 7016-20 2017.

[79] E. Balc, U. O. Akkuş, and S. Berber, "Band gap modification in doped MXene: Sc2CF2," J. Mater. Chem. C, vol. 5, pp. 5956-61, 2017.

[80] J. H. Sung, H. Heo, S. Si, Y. H. Kim, H. R. Noh, K. Song, J. Kim, C. S. Lee, S.-Y. Seo, D.-H. Kim, H. K. Kim, H. W. Yeom, T.-H. Kim, S.-Y. Choi, J. S. Kim, and M.-H. Jo, "Coplanar semiconductor-metal circuitry defined on few-layer MoTe2 via polymorphic heteroepitaxy," Nature Nanotechnology, 2017; doi:10.1038/nnano.2017.161

[81] Y. Matsuda, W.-Q. Deng, and W. A. Goddard III, "Contact resistance for "end-contacted" metal-graphene and metal-nanotube interfaces from quantum mechanics," J. Phys. Chem. C, vol. 114 (41), 17845$50,2010$.

[82] K. H. Khoo, W. S. Leong, J. T. L. Thong, and S. Y. Quek, "Origin of contact resistance at ferromagnetic metal-graphene interfaces," ACS Nano, vol. 10 (12), pp. 11219-27, 2016.

[83] M. Shaygan, M. Otto, A. A. Sagade, C. A. Chavarin, G. Bacher, W. Mertin, and D. Neumaier, "Low resistive edge contacts to CVDgrown graphene using a CMOS compatible metal," Annalen der Physik, 1600410, 2017.

[84] Y. Xu, C. Cheng, S. Du, J. Yang, B. Yu, J. Luo, W. Yin, E. Li, S. Dong, P. Ye, and X. Duan, "Contacts between two and threedimensional materials: Ohmic, Schottky, and p-n - heterojunctions,' ACS Nano, vol. 10 (5), pp. 4895-919, 2016.

[85] Y. Kim, A. R. Kim, J. H. Yang, K. E. Chang, J.-D. Kwon, S. Y. Choi, J. Park, K. E. Lee, D.-H. Kim, S. M. Choi, K. H. Lee, B. H. Lee, M G. Hahm, and B. Cho, "Alloyed 2D-metal-semiconductor heterojunctions: Origin of interface states reduction and Schottky barrier lowering," Nano Lett., vol. 16 (9), pp. 5928-33, 2016.

[86] 2-D Electronics' metal or semiconductor? Both, Researchers produced the first 2D field-effect transistor (FET) made of a single material, Science News, September, 2017, Text @ https://www.sciencedaily.com/releases /2017/09/ 170918161531. Htm

[87] J. H. Sung, H. Heo, S. Si, Y. H. Kim, H. R. Noh, K. Song, J. Kim, C. S. Lee, S.-Y. Seo, D.-H. Kim, H. K. Kim, H. W. Yeom, T.-H. Kim S.-Y. Choi, J. S. Kim, and M.-H. Jo, "Coplanar semiconductor-meta circuitry defined on few-layer MoTe2 via polymorphic heteroepitaxy," Nature Nanotechnology, 2017; DOI: 10.1038/NNANO.2017.161 